\title{
COMMUNITY ANALYSIS OF PLANT PARASITIC AND FREE LIVING NEMATODES ASSOCIATED WITH RICE AND SOYBEAN PLANTATION FROM PAKISTAN
}

\author{
MUSARRAT, A. R. ${ }^{* 1}-$ SHAHINA, F. ${ }^{2}-$ SHAH, A. A. ${ }^{1}-$ SABA, R. ${ }^{1}-$ FEROZA, K. ${ }^{2}$ \\ ${ }^{I}$ University of Sargodha sub campus Bhakkar, Bhakkar, Pakistan \\ ${ }^{2}$ National Nematological Research Centre University of Karachi, Pakistan \\ *Corresponding author \\ e-mail:marain79@gmail.com; phone:0332-3728140 \\ (Received 27 ${ }^{\text {th }}$ Apr 2016; accepted 22 $2^{\text {nd }}$ Jul 2016)
}

\begin{abstract}
Variety of nematode community indices have been proposed for purposes of environmental monitoring. During three phases of surveys (2005-08), thirty nine nematode genera other than root-knot nematodes were found associated with rice crop plants while 33 from soybean in the surveyed areas of rice and soybean. Nematodes were placed into five different groups (Yeates et al., 1993) viz., herbivores, fungivores, bacteriovores, omnivores and predators. In the present study highly abundant group is herbivores, followed by bacteriovores, fungivores, predators, and omnivores were encountered from rice and soybean fields. The occurrence percentage was observed in per $500 \mathrm{~g}$ soil for each nematode genus. All species of nematodes associated with soybean are first records of nematodes associated with soybean from Pakistan. Nematode communities were analyzed by mean abundance, prominence value, importance value and cluster analysis based on nematode numbers and presence or absence of nematode, respectively. Community relationship revealed the overall dominance of herbivores (Hirschmanniella and Helicotylenchus) and fungivores (Aphelenchoides) in rice and Tylenchorhynchus (herbivores), Aphelenchoides (fungivores), Panagrolaimus and Acrobelus (becteriovores) from soybean in all terms of community analysis. While the UPGMA cluster analysis showed that Multan and Dir localities exhibit close similarity coefficient in soybean and in rice Thatta and Sawat showed more similar coefficient or the nematode incidence
\end{abstract}

Keywords: cluster analysis, glycine max, nematodes, survey, nematode community

\section{Introduction}

Rice (Oriza sativa L.) is the staple food of more than two billion people, predominantly in Asia where more than $90 \%$ of the word's rice is grown and consumed (Bridge etal., 1990). Worldwide, rice yield losses due to plant parasitic nematodes are estimated at 10\% (Sasser and Freckman, 1987). The association of nematodes with rice in Punjab was reported by Anwar and Khan (1973) along with thirteen nematode genera including Hirschmanniella oryza and Radopholus oryza. Maqbool and Hashmi (1982) found plant parasitic nematodes in high frequency from rice growing areas of Pakistan. Maqbool (1983-84) reported Tylenchorhynchus rassicae, T. clarus, T. mashoodi and Basiria graminicola, respectively from soil around the roots of rice from Khyber Pakhtunkhawa (KP). Khan and Bilqees (1994) described a new species Basiria bajorensis from Bajore agency of paddy crops. Maqbool and Shahina (2001), was given biodiversity of nematode fauna of different Pakistani crops including rice. Musarrat et al. (2006) reported $M$. incognita from rice growing area of Sindh and KP, Pakistan. A review article on root knot nematode, Meloidogyne of Pakistan was given by Shahina et al. (2009). 
Soybean (Glycine max (L.) Merrill) is one of the most important oilseed crop in the world. The soybean cyst (Heterodera glycines) and root-knot (Meloidogyne spp.) nematodes have received the greatest emphasis in breeding program, contributing up to 4\% crop losses (Good, 1973). Maqbool (1981) in a general survey of crops reported the occurrence of root-knot nematode (M. hapla) on soybean. Severity of M. incognita on soybean was reported from Sindh and KP by Musarrat et al. (2006).More than 100 species of plant parasitic nematodes have been associated with soybean (Schmitt and Noel, 1984), the major pest species being Heterodera glycines, Meloidogyne spp., (M.incognita, M. arenaria and $M$. javanica) and Rotylenchulus reniformis. These nematodes are estimated to cause annual yield losses of over $10 \%$ to soybean on a worldwide basis (Sasser and Freckman, 1987). The aim of this study was to determine the percentage occurrence and community analysis of plant parasitic and free living soil nematodes.

\section{Material and methods}

\section{Survey and sample collection}

Extensive surveys were carried out from primary and secondary growing areas of rice and soybean from different agro ecological zones of Pakistan (except Balochistan) since 2005-2008. A total of 277 randomly chosen fields from 41 locations were visited and composite soil samples were taken from the rhizosphare of crop plants on each field (Table 1, Fig.1A \& B). in KPK area, zone 1 includes northern mountainous areas of the country and irrigated rice is grown either in flat valleys.the climate is sub-humid monsoon with 750-1000 mm average rainfall,mostly concentrated in summer. While in Punjab area, zone 2 lies in the broad strip of land between rivers Ravi and Chenab where both canal and sub soil water are used for irrigation.the climate is sub humid, sub-tropical type with 400 to $700 \mathrm{~mm}$ of rainfall mostly in July-August. In Sindh area, Zone 4 includes Indus delta which consists of vast spill flats and basins. The climate is arid tropical marine with no marked season.

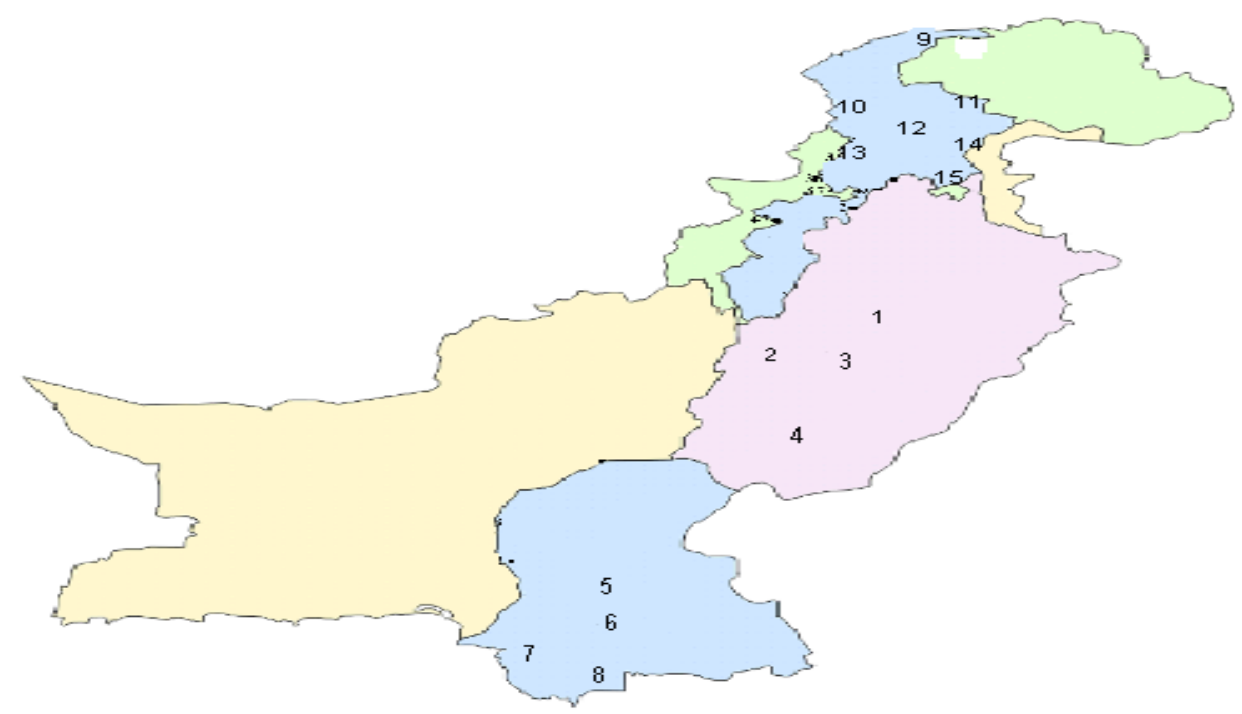

Figure 1A. Geographic localities of soybean growing areas of Pakistan.

Localities: 1-Faisalabad, 2-Multan, 3-Vehari, 4-Rahimyar Khan, 5-Sangharh, 6-Hyderabad, 7- Thatta, 8Badin, 9-Kurram Agency, 10-Dir, 11-Swat, 12-Mansehra, 13-Malakand, 14-Abbottabad, 15-Hazara. 


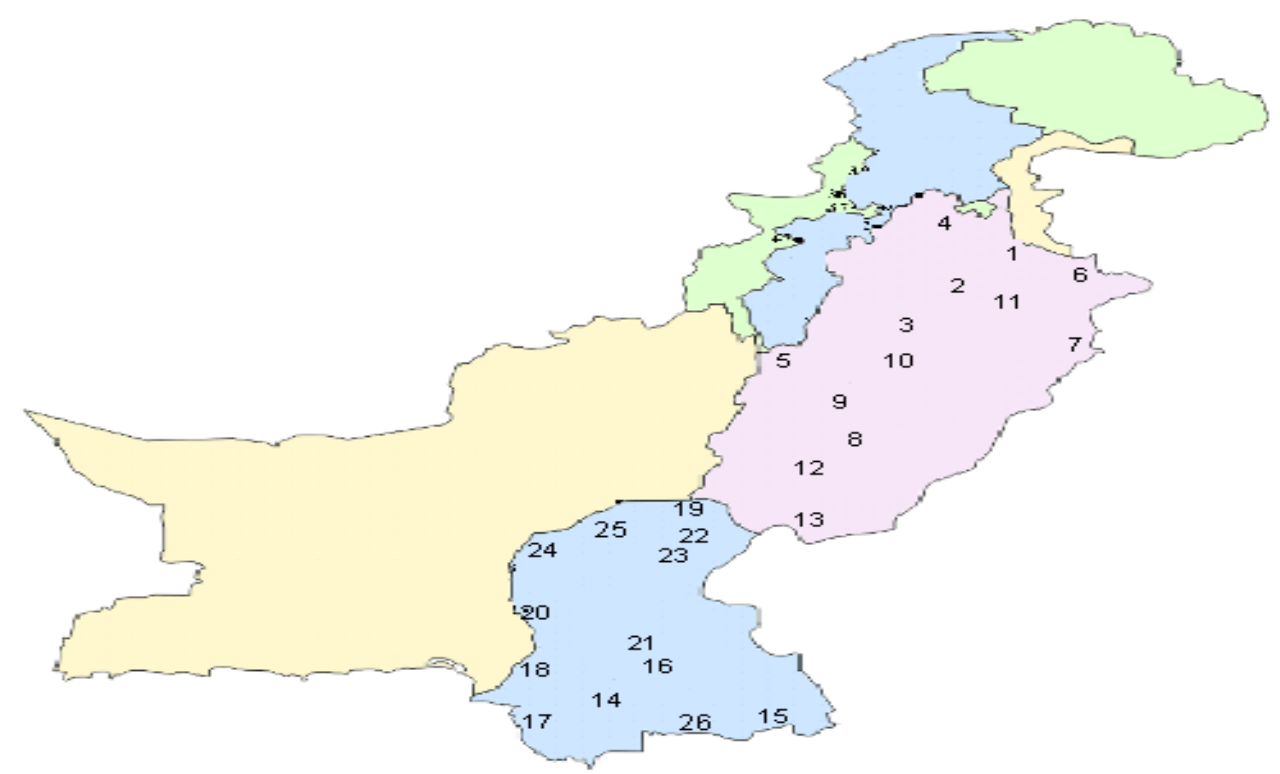

Figure 1B. Geographic localities of rice growing areas of Pakistan.

Localities: 1-Sialkot, 2-Gujranwala, 3-Sheikhupura, 4-Okara, 5-Jhang, 6-Narowal, 7-Kasur, 8-

Pakpattan, 9-Sahiwal, 10-Faisalabad, 11-Hafizabad, 12-Vehari, 13-Rahimyar Khan, 14-Hyderabad, 15Badin, 16-Sangharh, 17-Thatta, 18-Dadu, 19-Jaccobabad, 20-Larkana, 21-Nawabshah, 22-Sukkur, 23-

Khairpur, 24-Shikarpur, 25-Nasirabad, 26- Gharo.

\section{Extraction of nematodes}

Extraction of plant parasitic and free living nematodes from soil was made by Cobb's sieving and Baermann funnel techniques (Baermann, 1917 and Cobb, 1918, respectively).

\section{Quantitative analysis}

For the community analysis nematodes were counted in an open counting chamber with only $5 \mathrm{ml}$ extracted nematode suspension by a counter under binocular microscope. Process was repeated 3 times and average of three readings gave the number of nematodes per unit of soil.

\section{Data analysis}

Community analysis of phytoparasitic and free living nematodes in rice and soybean fields of Pakistan was done by the using of Norton techniques (1978).

The similarity matrix based on the quantitative analysis (presence (1)/absence $(0)$ of nematodes, which used to establish the similarity between localities on the basis of Jacord's coefficient of similarity (Rohlf, 2005). Dandrogram constructed on the basis of data was related to the localities. All computations were carried out using the NTsys packages, version 2.2 (Rohlf, 2005). 
Table 1. Surveyed localities of rice and soybean of Pakistan.

\begin{tabular}{|c|c|c|c|c|c|c|c|}
\hline S.\# & $\begin{array}{l}\text { Localities of } \\
\text { rice }\end{array}$ & Latitude & Longitude & S.\# & $\begin{array}{c}\text { Localities of } \\
\text { soybean }\end{array}$ & Latitude & Longitude \\
\hline 1 & Rahimyar Khan & $28^{\circ} 30^{\prime} \mathrm{N}$ & $70^{\circ} 25^{`} \mathrm{E}$ & 1 & Multan & $30^{\circ} 15^{\prime} \mathrm{N}$ & $71^{\circ} 36^{\prime} \mathrm{E}$ \\
\hline 2 & Sialkot & $32^{\circ} 30^{\prime} \mathrm{N}$ & $74^{\circ} 31^{`} \mathrm{E}$ & 2 & Vehari & $29^{\circ} 15^{\top} \mathrm{N}$ & $71^{\circ} 30^{\circ} \mathrm{E}$ \\
\hline 3 & Hafizabad & $32^{\circ} 05^{`} \mathrm{~N}$ & $73^{\circ} 40^{\prime} \mathrm{E}$ & 3 & Faisalabad & $31^{\circ} 25^{\prime} \mathrm{N}$ & $73^{\circ} 09^{`} \mathrm{E}$ \\
\hline 4 & Sialkot & $32^{\circ} 15^{\top} \mathrm{N}$ & $74^{\circ} 52^{`} \mathrm{E}$ & 4 & Rahimyar Khan & $28^{\circ} 30^{\circ} \mathrm{N}$ & $70^{\circ} 25^{`} \mathrm{E}$ \\
\hline 5 & Gujranwala & $32^{\circ} 10^{`} \mathrm{~N}$ & $74^{\circ} 12^{`} \mathrm{E}$ & 5 & Hyderabad & $25^{\circ} 23^{\prime} \mathrm{N}$ & $68^{\circ} 24^{\prime} \mathrm{E}$ \\
\hline 6 & Narowal & $32^{\circ} 06^{`} \mathrm{~N}$ & $74^{\circ} 52^{`} \mathrm{E}$ & 6 & Badin & $24^{\circ} 38^{\prime} \mathrm{N}$ & $68^{\circ} 54^{`} \mathrm{E}$ \\
\hline 7 & Jhang & $31^{\circ} 15^{`} \mathrm{~N}$ & $74^{\circ} 22^{`} \mathrm{E}$ & 7 & Sangharh & $26^{\circ} 20^{\prime} \mathrm{N}$ & $68^{\circ} 57^{`} \mathrm{E}$ \\
\hline 8 & Sheikhupura & $30^{\circ} 32^{`} \mathrm{~N}$ & $71^{\circ} 80^{\circ} \mathrm{E}$ & 8 & Thatta & $33^{\circ} 35^{\prime} \mathrm{N}$ & $74^{\circ} 14^{\prime} \mathrm{E}$ \\
\hline 9 & Sahiwal & $30^{\circ} 45^{\prime} \mathrm{N}$ & $73^{\circ} 80^{`} \mathrm{E}$ & 9 & Hazara & $33^{\circ} 59^{\circ} \mathrm{N}$ & $72^{\circ} 56^{\circ} \mathrm{E}$ \\
\hline 10 & Okara & $30^{\circ} 50^{`} \mathrm{~N}$ & $73^{\circ} 31^{\prime} \mathrm{E}$ & 10 & Swat & $34^{\circ} 40^{\prime} \mathrm{N}$ & $72^{\circ} 52^{`} \mathrm{E}$ \\
\hline 11 & Vehari & $29^{\circ} 15^{`} \mathrm{~N}$ & $71^{\circ} 30^{`} \mathrm{E}$ & 11 & Dir & $35^{\circ} 12^{\prime} \mathrm{N}$ & $71^{\circ} 53^{`} \mathrm{E}$ \\
\hline 12 & Kasur & $31^{\circ} 07^{\top} \mathrm{N}$ & $74^{\circ} 27^{`} \mathrm{E}$ & 12 & Kurram Agency & $34^{\circ} 40^{\prime} \mathrm{N}$ & $71^{\circ} 55^{`} \mathrm{E}$ \\
\hline 13 & Pakpattan & $31^{\circ} 21^{\prime} \mathrm{N}$ & $73^{\circ} 24^{`} \mathrm{E}$ & 13 & Mansehra & $34^{\circ} 25^{\prime} \mathrm{N}$ & $71^{\circ} 50^{\circ} \mathrm{E}$ \\
\hline 14 & Faisalabad & $31^{\circ} 25^{\prime} \mathrm{N}$ & $73^{\circ} 09^{`} \mathrm{E}$ & 14 & Malakand Agency & $34^{\circ} 40^{\prime} \mathrm{N}$ & $71^{\circ} 55^{`} \mathrm{E}$ \\
\hline 15 & Nawabshah & $26^{\circ} 15^{\top} \mathrm{N}$ & $68^{\circ} 25^{\circ} \mathrm{E}$ & 15 & Abbottabad & $34^{\circ} 26^{\prime} \mathrm{N}$ & $71^{\circ} 52^{`} \mathrm{E}$ \\
\hline 16 & Sukkur & $28^{\circ} 55^{`} \mathrm{~N}$ & $68^{\circ} 55^{\circ} \mathrm{E}$ & & & & \\
\hline 17 & Khairpur & $27^{\circ} 06^{`} \mathrm{~N}$ & $87^{\circ} 44^{\top} \mathrm{E}$ & & & & \\
\hline 18 & Sangharh & $26^{\circ} 20^{\prime} \mathrm{N}$ & $68^{\circ} 57^{`} \mathrm{E}$ & & & & \\
\hline 19 & Hyderabad & $25^{\circ} 23^{\prime} \mathrm{N}$ & $68^{\circ} 24^{`} \mathrm{E}$ & & & & \\
\hline 20 & Badin & $24^{\circ} 38^{\prime} \mathrm{N}$ & $68^{\circ} 54^{`} \mathrm{E}$ & & & & \\
\hline 21 & Thatta & $33^{\circ} 35^{\prime} \mathrm{N}$ & $74^{\circ} 14^{\prime} \mathrm{E}$ & & & & \\
\hline 22 & Dadu & $26^{\circ} 06^{`} \mathrm{~N}$ & $67^{\circ} 45^{`} \mathrm{E}$ & & & & \\
\hline 23 & Jaccobabad & $28^{\circ} 17^{\prime} \mathrm{N}$ & $68^{\circ} 26^{`} \mathrm{E}$ & & & & \\
\hline 24 & Larkana & $27^{\circ} 32^{`} \mathrm{~N}$ & $68^{\circ} 18^{`} \mathrm{E}$ & & & & \\
\hline 25 & Nasirabad & $27^{\circ} 32^{`} \mathrm{~N}$ & $69^{\circ} 18^{`} \mathrm{E}$ & & & & \\
\hline 26 & Gharo & $24^{\circ} 44^{\prime} \mathrm{N}$ & $67^{\circ} 35^{`} \mathrm{E}$ & & & & \\
\hline
\end{tabular}

\section{Results}

\section{Percentage occurrence of nematode genera in rice plantations}

The nematode genera encountered from rice plantations were identified and characterized into different groups as follows: Out of 39 nematode genera 15 belongs to harbivores viz., (Basiria, Boleodorus, Criconemoides, Ditylenchus, Helicotylenchus, Heterodera, Hirschmanniella, Hoplolaimus, Longidorus, Merlinius, Paratylenchus, Pratylenchus, Psilenchus, Tylenchorhynchus and Xiphinema); while four genera (Aphelenchoides, Aphelenchus, Filenchus and Dorylaimus) belong to fungivores. Six genera (Clarkus, Ironus, Laimydorus, Mononchus, Mylonchulus and Oinchus); and two genera (Eudorylaimus and Mesodorylaimus) of predators and omnivores were encountered respectively. Among these nematodes the second highest genera were bacteriovores (Acrobelus, Cephalobus, Mesorhabditis, Diplogaster, Diploscapter, Discolaimium, Eucephalobus, Geomonhystera, Panagrolaimus, Plectus, Rhabditis and Zeldia).

The most frequently occurring nematode genera were Hirschmanniella, Aphelenchoides, Helicotylenchus and Tylenchorhynchus from rice cultivated areas 
which were surveyed. These nematodes were found in relatively high densities as compare to other plant parasitic nematodes from surveyed areas of both provinces (Table 2).

Table 2. Occurrence (percentage) of nematode genera regarding their diverse groups from rice fields of Pakistan during 2005-08.

\begin{tabular}{|c|c|c|c|c|c|c|}
\hline \multirow{2}{*}{$\begin{array}{l}\text { Trophic groups of } \\
\text { nematode }\end{array}$} & \multicolumn{3}{|c|}{ Punjab } & \multicolumn{3}{|c|}{ Sindh } \\
\hline & 2005-06 & 2006-07 & 2007-08 & 2005-06 & 2006-07 & 2007-08 \\
\hline \multicolumn{7}{|c|}{ Herbivores nematodes } \\
\hline Basiria & 3 & 4 & 1 & 2 & 3 & 0.99 \\
\hline Boleodorus & 1 & - & - & 2.5 & 2 & - \\
\hline Criconemoides & 1 & 0.9 & - & - & - & 1 \\
\hline Ditylenchus & 10 & 12 & 9.8 & 5 & 3.9 & 6.3 \\
\hline Helicotylenchus & 60 & 62 & 55 & 72 & 66 & 58 \\
\hline Heterodera & 1 & - & - & - & - & - \\
\hline Hirschmanniella & 66 & 58 & 62 & 90 & 95 & 88 \\
\hline Hoplolaimus & 5 & 4 & 3 & 1 & 2.5 & 1.5 \\
\hline Longidorus & 1 & - & - & 0.9 & 0.4 & - \\
\hline Merlinius & 1 & - & 1.5 & 2 & 1.5 & 0.9 \\
\hline Paratylenchus & 3.8 & 6 & 3 & 2 & 1 & 6.3 \\
\hline Pratylenchus & 1 & 0.9 & 1 & 0.8 & 1.5 & 0.44 \\
\hline Psilenchus & 1 & - & - & 1 & 0.9 & 1 \\
\hline Tylenchorhynchus & 20 & 15 & 11 & 25 & 45 & 35 \\
\hline Xiphinema & 2 & 1 & 3 & 1 & - & - \\
\hline \multicolumn{7}{|l|}{ Fungivores } \\
\hline Aphelenchoides & 60 & 45 & 73 & 70 & 71 & 62 \\
\hline Aphelenchus & 32 & 30 & 18 & 25 & 40 & 20 \\
\hline Dorylaimus & 2 & 1 & 3 & 3.3 & 4 & 2 \\
\hline Filenchus & 1 & 0.9 & 0.8 & - & - & 1 \\
\hline \multicolumn{7}{|l|}{ Bacteriovores } \\
\hline Acrobelus & 25 & 20 & 18 & 40 & 39 & 33.2 \\
\hline Cephalobus & 15 & 25 & 33 & 60 & 65 & 70 \\
\hline Mesorhabditis & 20 & 15 & 23 & 35 & 38 & 33 \\
\hline Diplogaster & 13 & 15 & 20 & 60 & 55 & 51 \\
\hline Diploscapter & 8 & 10 & 7 & 15 & 13 & 11 \\
\hline Discolaimium & 6 & 4 & 3 & 10 & 12 & 18 \\
\hline Eucephalobus & 2 & 2.5 & 1 & 3 & 5 & 6.5 \\
\hline Geomonhystera & 15 & 20 & 22 & 10 & 11 & 13 \\
\hline Panagrolaimus & 18 & 11 & 19 & 23 & 42 & 40 \\
\hline Plectus & 8 & 6 & 4 & 25 & 33 & 26 \\
\hline Rhabditis & 15 & 13 & 10 & 8 & 6.5 & 10 \\
\hline Zeldia & 15 & 22 & 23 & 11 & 13 & 20 \\
\hline \multicolumn{7}{|l|}{ Omnivores } \\
\hline Eudorylaimus & 1 & - & - & 3.5 & 4 & 2.5 \\
\hline Mesodorylaimus & 3 & 1 & 1 & 1 & 2 & 1 \\
\hline \multicolumn{7}{|l|}{ Predators } \\
\hline Clarkus & 55 & 50 & 45 & 65 & 66 & 62 \\
\hline Ironus & 1 & 1 & - & 0.8 & 0.6 & 1 \\
\hline Laimydorus & 8 & 6 & 10 & 30 & 10 & 28 \\
\hline Mononchus & - & - & 1 & 0.9 & 2 & 1 \\
\hline Mylonchulus & 10 & 8 & 7 & 22 & 25 & 33 \\
\hline Oinchus & 1 & - & - & 2 & 1 & - \\
\hline
\end{tabular}


The samples contained genera of diverse group with a range of 5 to $39 \%$. Studies on $\%$ occurrence of these groups revealed that herbivores dominated the entire nematode community in \% occurrence (39\%) followed by bacteriovores $(31 \%)$, predators $(15 \%)$, fungivores (10\%) and omnivores (5\%) (Fig. 2A).

In terms of overall \% occurrence of nematode genera among five different groups, Hirschmanniella (herbivores) was most frequently occurred genus with the highest \% occurrence (21\%), followed by Aphelenchoides (fungivores) and Helicotylenchus (herbivores) sharing the same \% occurrence (17\%). The second highly abundance nematode genus was predator Clarkus (16\%) followed by bacteriovores group with Cephalobus (16\%), Diplogaster (10\%) and Panagrolaimus (7\%) in the surveyed areas of rice during three years (2005-2008) (Fig. 2B).
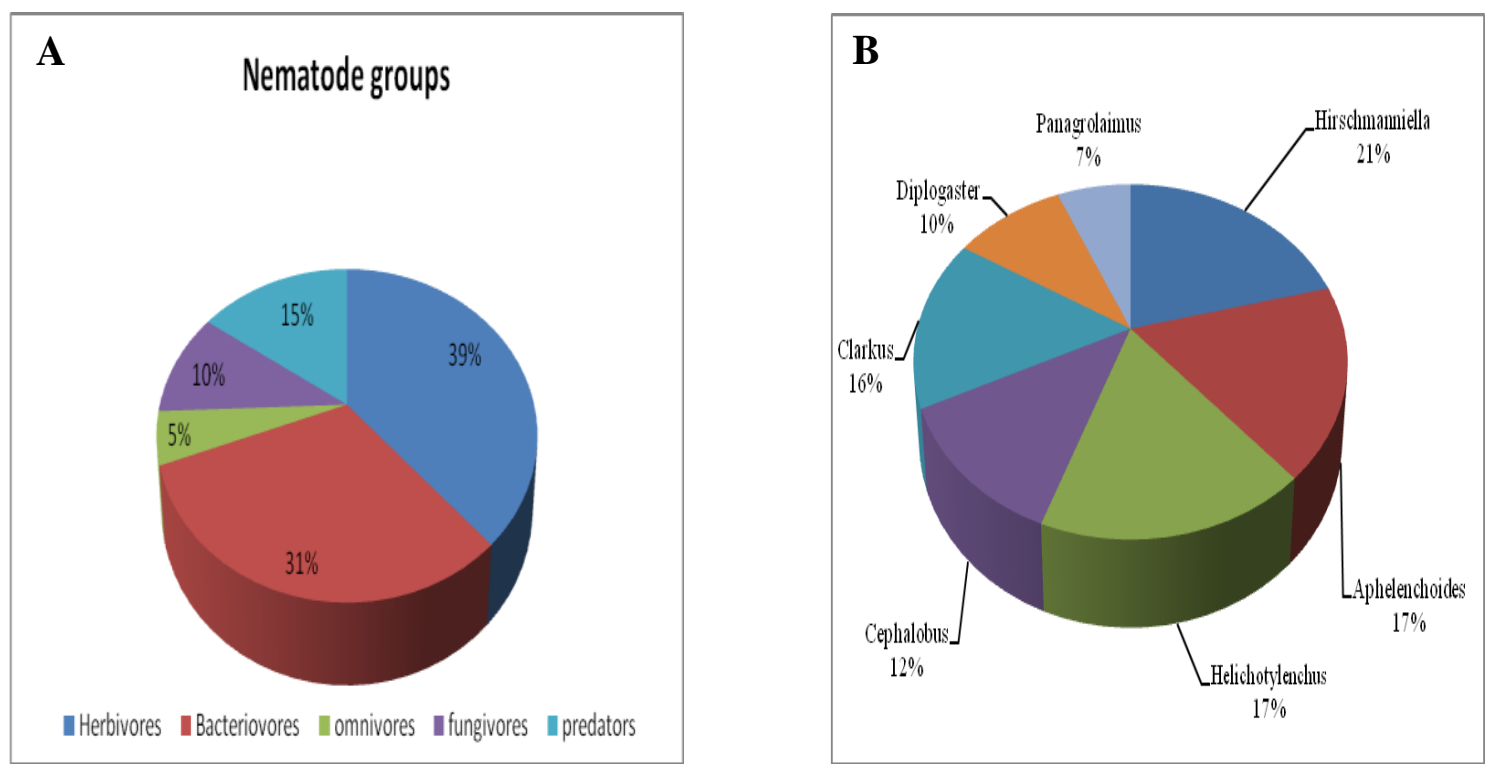

Figure 2. A. Different groups of nematodes in rice; B. Occurrence (\%) of nematode genera in rice surveyed areas.

\section{Percentage occurrence of nematode genera in soybean plantations}

Soil analysis revealed the presence of 33 nematode genera encountered from soybean crop plantations during 2005 to 2008 surveys from Sindh, Punjab and KP (Table. 3A-B; Fig. 3A-B). These genera belonged to five diverse groups in which herbivores contain 15 nematode genera, fungivores with 6 genera; bacteriovores have 10 genera; predators contain 3 genera and only one genus representing the omnivores. Among these five groups herbivores dominated with the highest percentage occurrence $(40 \%)$, followed by bacteriovores $(30 \%)$, fungivores $(18 \%)$, predators $(9 \%)$ and omnivores $(3 \%)$ in the nematode community structure (Fig. 3A).

In terms of overall \% occurrence of nematode genera, Helicotylenchus (29\%) was the most occurred (herbivores) genus whereas Tylenchorhynchus (herbivores, 17\%) followed by Aphelenchoides (fungivores, 12\%) were encountered in abundance from all fields of soybean crop .However other nematode groups such as bacteriovores: Panagrolaimus (18\%), Plectus (14\%) and Acrobelus (10\%) frequently encountered from soybean soil samples (Fig. 3B). 


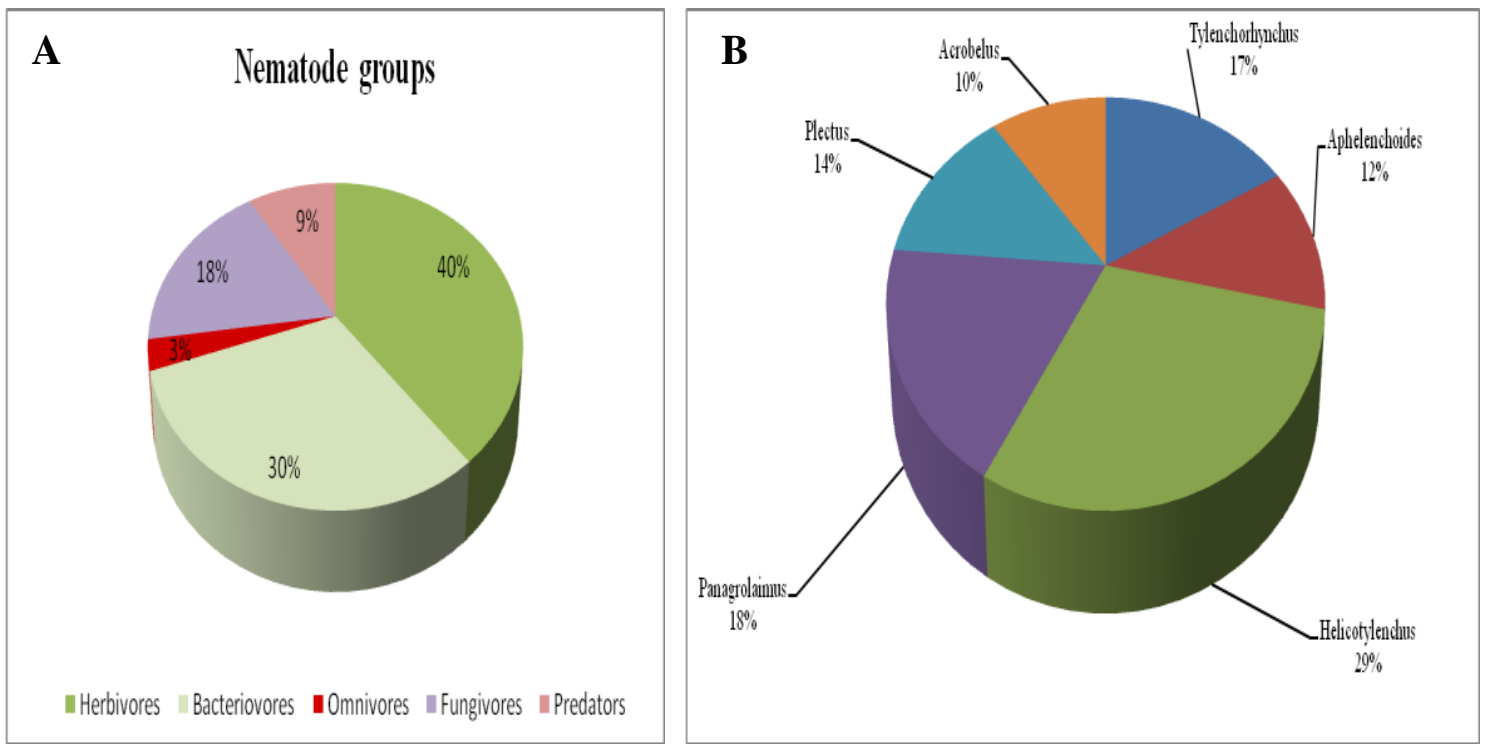

Figure 3. A. Different groups of nematodes from soybean; B. \% occurrence of nematode genera in soybean surveyed areas.

Table 3A. Percentage occurrence of nematode genera regarding their diverse groups from soybean fields of Pakistan during 2005-08.

\begin{tabular}{|c|c|c|c|c|c|c|c|c|c|}
\hline \multirow{2}{*}{$\begin{array}{c}\text { Nematode genera } \\
\text { Herbivores }\end{array}$} & \multicolumn{3}{|c|}{ Punjab } & \multicolumn{3}{|c|}{ Sindh } & \multicolumn{3}{|c|}{$\begin{array}{c}\text { Khyber } \\
\text { Pakhtunkhwa }\end{array}$} \\
\hline & 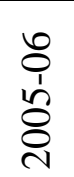 & $\begin{array}{l}\text { ô } \\
\vdots \\
8 \\
\varnothing\end{array}$ & 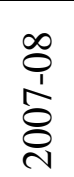 & 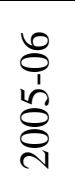 & 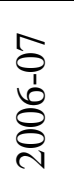 & 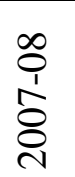 & 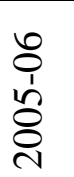 & 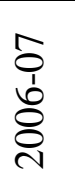 & 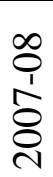 \\
\hline Boleodorus spp. & 2 & 1 & 1 & 1 & 0.4 & - & 1 & - & - \\
\hline Criconemoides spp. & 1 & 1 & 4 & 3.5 & 1 & 2 & 1 & 2.5 & 2 \\
\hline Ditylenchus spp. & 7 & 10 & 10 & 8 & 5 & 3 & - & 2 & - \\
\hline Gracilacus spp. & - & - & - & 1 & 0 & 2 & - & - & - \\
\hline Helicotylenchus spp. & 36 & 60 & 53 & 42 & 55 & 63 & 41 & 33 & 52 \\
\hline Heterodera spp. & 0.5 & 1 & - & - & - & - & - & - & - \\
\hline Hoplolaimus spp. & 0.6 & 1 & - & 2 & 1 & 5 & 4 & - & - \\
\hline Longidorus spp. & 0.2 & 1 & - & - & - & 1 & 0.4 & 0.2 & - \\
\hline Neodolichorhynchus spp. & - & - & - & 3 & 5 & - & 2 & 5 & 3 \\
\hline Paratylenchus spp. & 1 & 6 & 8 & 4 & 7 & 8 & 3 & 6 & 4 \\
\hline Psilenchus spp. & 0.2 & 1 & 0.6 & - & - & 1 & 1 & - & - \\
\hline Tylenchorhynchus spp. & 25 & 35 & 30 & 29 & 28 & 26 & 22 & 24 & 30 \\
\hline Xiphinema spp. & 5 & 9 & 10 & 3 & 4 & 1 & 3 & 2 & - \\
\hline \multicolumn{10}{|c|}{ Fungivores } \\
\hline Aphelenchoides spp. & 25 & 30 & 15 & 20 & 22 & 28 & 15 & 16 & - \\
\hline
\end{tabular}




\begin{tabular}{|c|c|c|c|c|c|c|c|c|c|}
\hline Aphelenchus spp. & 15 & 20 & 30 & 23 & 34 & 37 & 20 & 10 & 10 \\
\hline Dorylaimus spp. & 15 & 13 & 10 & 9 & 7 & 3 & 2 & - & 1 \\
\hline Discolaimus spp. & 1 & 2 & 1 & 2 & 3 & 1 & 1 & 1.5 & - \\
\hline Filenchus spp. & 4 & 3.6 & 5 & 3 & 2 & 1 & 1 & 3 & 1 \\
\hline Nygolaimus spp. & 1 & 1.5 & - & 1 & 1 & 1 & - & - & - \\
\hline
\end{tabular}

Table 3B. Percentage occurrence of diverse groups of nematodes from soybean fields of Pakistan during 2005-08.

\begin{tabular}{|c|c|c|c|c|c|c|c|c|c|}
\hline \multirow[t]{2}{*}{ Nematode genera } & \multicolumn{3}{|c|}{ Punjab } & \multicolumn{3}{|c|}{ Sindh } & \multicolumn{3}{|c|}{$\begin{array}{c}\text { Khyber } \\
\text { Pakhtunkhwa }\end{array}$} \\
\hline & 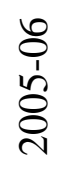 & 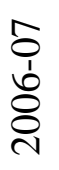 & 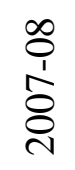 & 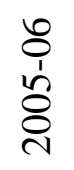 & 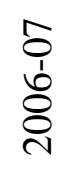 & 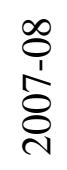 & 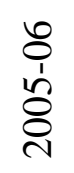 & 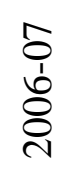 & 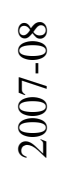 \\
\hline \multicolumn{10}{|l|}{ Bacteriovores } \\
\hline Acrobelus spp. & 12 & 10 & 19 & 25 & 36 & 30 & 8 & 6 & 5 \\
\hline Cephalobus spp. & 30 & 25 & 20 & 15 & 19 & 20 & 15 & 13 & 12 \\
\hline Diplogaster spp. & 5 & 8 & 20 & 15 & 14 & 18 & 6 & 8 & 3 \\
\hline Diploscapter spp. & 1 & 0 & 1 & 0.4 & 0.5 & - & 1 & - & - \\
\hline $\begin{array}{l}\text { Mesodorylaimus } \\
\text { spp. }\end{array}$ & 8 & 6 & 7 & 4 & 3 & 5 & 1 & - & - \\
\hline $\begin{array}{l}\text { Panagrolaimus } \\
\text { spp. }\end{array}$ & 40 & 35 & 55 & 35 & 30 & 29 & 12 & 18 & 19 \\
\hline Plectus spp. & 18 & 22 & 15 & 35 & 25 & 40 & 20 & 15 & 10 \\
\hline Rhabditids spp. & 7 & 5 & 6.8 & 3 & 5 & 2 & 1 & 1.5 & - \\
\hline Rhabdolaimus spp. & 3 & 2 & 1 & 1 & 3 & 4 & 1 & - & - \\
\hline Seleborca & 1 & 2 & 1 & 0 & 0 & 1 & 2 & 1 & 1 \\
\hline \multicolumn{10}{|l|}{ Predators } \\
\hline Laimydorus spp. & 1 & 1 & - & - & - & 1 & - & - & - \\
\hline Mononchus spp. & 5 & 6 & 8 & 2 & 4 & 6 & 1 & 3 & 5 \\
\hline Mylonchulus spp. & 6 & 3 & 1 & 5 & 6 & 4 & 2 & 1 & 2 \\
\hline \multicolumn{10}{|l|}{ Omnivores } \\
\hline Eudorylaimus spp. & - & 5 & 2 & 8 & 10 & 15 & 4 & 2 & - \\
\hline
\end{tabular}

\section{Community analysis of nematode genera in rice fields}

To exhibit the relationship of nematodes associated with host plants the community analysis was performed which provides information concerning frequency (absolute and relative frequency) and density (absolute and relative density) of nematodes on studied plant hosts. 
Community relationship revealed the overall dominance of herbivores (Hirschmanniella and Helicotylenchus) and fungivores (Aphelenchoides) in all terms of community analysis (Table 4).

Table 4. Community analysis of plant parasitic and free-living soil nematodes in rice growing areas of Pakistan.

\begin{tabular}{|c|c|c|c|c|c|}
\hline Nematode genera & A.F & R.F & A.D & R.D & P.V \\
\hline \multicolumn{6}{|l|}{ Plant parasitic nematodes } \\
\hline Aphelenchoides spp. & 27.9 & 6.2 & 233 & 5.9 & 16.7 \\
\hline Aphelenchus spp. & 17.3 & 4.6 & 107 & 4.9 & 10.5 \\
\hline Basiria spp. & 4.2 & 1.4 & 22 & 1.2 & 1.6 \\
\hline Boleodorus spp. & 3.4 & 1.0 & 25 & 1.5 & 1.2 \\
\hline Criconemoides spp. & 3.2 & 1.0 & 24 & 1.6 & 1.8 \\
\hline Ditylenchus spp. & 3.8 & 1.8 & 66 & 3.3 & 3.2 \\
\hline Filenchus spp. & 1.5 & 0.41 & 66 & 3.5 & 2.9 \\
\hline Helicotylenchus spp. & 23 & 6.1 & 121 & 5.5 & 13.9 \\
\hline Heterodera spp. & 3.6 & 2.0 & 8.0 & 2.1 & 2.0 \\
\hline Hirschmanniella spp. & 66.2 & 12.8 & 253 & 9.8 & 33 \\
\hline Hoplolaimus spp. & 15.3 & 5.1 & 110 & 4.9 & 1.2 \\
\hline Longidorous spp. & 4.3 & 1.5 & 20 & 1.2 & 1.5 \\
\hline Merlinius spp. & 1.6 & 0.51 & 39 & 1.6 & 1.14 \\
\hline Paratylenchus spp. & 2.1 & 0.21 & 22 & 0.6 & 1.0 \\
\hline Pratylenchus & 1.5 & 0.5 & 32 & 1.5 & 1.12 \\
\hline Psilenchus spp. & 1.6 & 0.3 & 55 & 2.3 & 1.8 \\
\hline Tylenchorhynchus spp. & 41.3 & 12.8 & 223 & 9.4 & 31 \\
\hline Xiphinema spp. & 3.7 & 1.0 & 28 & 1.4 & 1.42 \\
\hline \multicolumn{6}{|l|}{ Free- living soil nematodes } \\
\hline Acrobelus spp. & 23.5 & 5.9 & 326 & 5.8 & 4.2 \\
\hline Cephalobus spp. & 16.4 & 6.2 & 255 & 4.7 & 3.2 \\
\hline Cervidellus spp. & 11.2 & 2.3 & 99 & 0.36 & 3.2 \\
\hline Clarkus & 1.3 & 0.5 & 66 & 3.4 & 2.6 \\
\hline Diplogaster spp. & 9.6 & 6.4 & 110 & 5.3 & 3.5 \\
\hline Diploscapter spp. & 8.5 & 3.2 & 88 & 2.1 & 1.9 \\
\hline Discolaimium spp. & 9.5 & 2.4 & 49 & 2.1 & 3.1 \\
\hline Dorylaimus spp. & 1.9 & 0.34 & 26 & 1.3 & 2.8 \\
\hline Eucephalobus spp. & 5.6 & 0.6 & 44 & 1.3 & 1.5 \\
\hline Eudorylaimus spp. & 1.3 & 0.54 & 20 & 1.6 & 2.1 \\
\hline Geomonhystera spp. & 9.8 & 1.2 & 33 & 2.3 & 2.3 \\
\hline Ironus spp. & 8.5 & 2.4 & 22 & 1.6 & 1.3 \\
\hline Laimydorus spp. & 1.1 & 0.5 & 15 & 1.32 & 2.4 \\
\hline Mesodorylaimus & 8.3 & 3.1 & 79 & 2.0 & 1.8 \\
\hline Mononchus spp. & 5.4 & 1.5 & 110 & 5.0 & 6.3 \\
\hline Mylonchulus & 4.6 & 1.4 & 98.5 & 4.8 & 5.9 \\
\hline Oinchus spp. & 3.2 & 0.4 & 9 & 1.0 & 1.1 \\
\hline Panagrolaimus spp. & 14.2 & 5.6 & 99 & 2.3 & 2.1 \\
\hline Plectus spp. & 25.3 & 5.8 & 206 & 5.6 & 7.3 \\
\hline Rhabditis spp. & 11.3 & 2.5 & 76 & 3.3 & 2.6 \\
\hline Zeldia spp. & 5.6 & 0.5 & 45 & 2.6 & 1.3 \\
\hline
\end{tabular}

Absolute frequency (A.F) $=($ No of samples containing a species / Total samples collected $) 100$; Relative frequency $($ R.F $)=($ Frequency of a species in a sample/ Sum of frequencies of all species present $) 100$, Absolute density (A.D) $=$ (No of individuals of a species in a sample/ volume of the sample) 100; Relative density (R.D) $=$ No of individuals of a species in a sample / Total no. of individuals in the sample ) 100 ; Prominence value (P.V) $=($ Absolute density/ Absolute frequency). 


\section{Frequency}

Hirschmanniella $(\mathrm{AF}=66.2 \%)$ was the most prevalent genus in the entire plant parasitic nematode community, followed by Tylenchorhynchus $(\mathrm{AF}=41.3 \%)$; Aphelenchoides $(\mathrm{AF}=27.9 \%)$ and Helicotylenchus $(\mathrm{AF}=23.0 \%)$, while the least prevalent genus was Filenchus $(\mathrm{AF}=1.5 \%)$.

Among the free-living soil nematodes, Plectus $(\mathrm{AF}=25.3 \%)$ and Acrobelus $(\mathrm{AF}=$ $23.5 \%)$ were the most prevalent genera. Laimydorus $(\mathrm{AF}=1.1 \%)$ was the least prevalent genus in rice fields (Table 4).

\section{Absolute density}

Among all the nematode genera recorded, Hirschmanniella had maximum absolute density $(\mathrm{AD}=253 / \mathrm{sample})$, followed by Aphelenchoides $(\mathrm{AD}=233 / \mathrm{sample})$ and Tylenchorhynchus $(\mathrm{AD}=223$ / sample), while the genus Longidorus had lowest absolute density $(\mathrm{AD}=20.0 /$ sample $)$. In free-living soil nematode group studies, Acrobelus had the highest absolute density $(\mathrm{AD}=326 /$ sample) followed by Cephalobus ( $\mathrm{AD}=255$ / sample) and Plectus $(\mathrm{AD}=206 /$ sample), while Oinchus had the lowest mean density 9 per sample.

\section{Prominence value}

The rice nematode genus Hirschmanniella had the highest prominence value ( $\mathrm{PV}=$ 33.0), followed by Tylenchorhynchus ( $\mathrm{PV}=31.0)$, while the least $\mathrm{PV}$ was found in genus Paratylenchus $(\mathrm{PV}=1.0)$. Among the free-living soil nematodes Plectus had maximum PV (7.3), followed by Mononchus ( $\mathrm{PV}=6.3$ ), while the least prominence value was recorded in Oinchus $(\mathrm{PV}=1.1)$.

\section{Community analysis of nematode genera in soybean plantations}

Detailed analysis of different parameters for nematode community analysis has been provided in the Table 5 for the soybean crop.

\section{Frequency}

Among the plant parasites, the most frequently encountered genus was Tylenchorhynchus $(\mathrm{AF}=45.8 \%)$; followed by Aphelenchoides $(\mathrm{AF}=45.3 \%)$, whereas Boleodorus was least frequent $(\mathrm{AF}=1.3 \%)$ genus. Genus Panagrolaimus and Acrobelus were the most prevalent genera among the free-living soil nematodes with absolute frequencies (AF) $40 \%$ and 36\%, respectively whereas the least frequent genus was Diploscapter $(\mathrm{AF}=1.5 \%)$.

\section{Absolute density}

Genus Tylenchorhynchus had the highest absolute density $(\mathrm{AD}=222)$, followed by Aphelenchoides $(\mathrm{AD}=205)$ while the least density was of the genus Gracilacus $(\mathrm{AD}=12)$. Among the free-living soil nematodes Panagrolaimus and Acrobelus were the most dominant genera in the entire nematode community in soybean fields with $\mathrm{AD}=253$ and $\mathrm{AD}=221$, respectively. Whereas Diploscapter was the least dominant genus with $\mathrm{AD}=22$. 


\section{Prominence value}

Community analysis of plant parasitic nematodes associated with soybean crop revealed that highest prominence value of Tylenchorhynchus $(\mathrm{PV}=32)$ and Aphelenchoides was noted as second most prevalent genus with $\mathrm{PV}=31$, while the least prominence values (PV=1.1 and PV=1.2) were recorded for Filenchus and Longidorus genera respectively.

In case of free-living soil nematodes maximum prominence value was recorded in genus Acrobelus (PV=33), while the least prominence value ( $\mathrm{PV}=1.2)$ was recorded in the genus Diploscapter (Table 5).

Table 5. Community analysis of plant parasitic and free-living soil nematodes in soybean growing areas of Pakistan.

\begin{tabular}{|c|c|c|c|c|c|}
\hline Nematode genera & A.F & R.F & A.D & R.D & P.V \\
\hline \multicolumn{6}{|c|}{ Plant parasitic nematodes } \\
\hline Aphelenchoides & 45.3 & 12.4 & 205 & 9.4 & 31 \\
\hline Aphelenchus & 15.3 & 5.5 & 106 & 4.8 & 10.5 \\
\hline Boleodorus & 1.3 & 0.51 & 55 & 2.5 & 1.7 \\
\hline Criconemoides & 3.8 & 1.3 & 38 & 1.6 & 1.6 \\
\hline Ditylenchus & 3.7 & 1.0 & 33 & 1.4 & 1.4 \\
\hline Filenchus & 1.9 & 0.5 & 35 & 1.5 & 1.1 \\
\hline Gracilacus & 1.6 & 0.21 & 12 & 2.1 & 1.6 \\
\hline Helicotylenchus & 20 & 7.3 & 109 & 5.5 & 9.8 \\
\hline Heterodera & 5.5 & 1.5 & 44 & 5.0 & 6.2 \\
\hline Hoplolaimus & 3.8 & 1.0 & 31 & 1.4 & 1.3 \\
\hline Longidorous & 1.9 & 0.5 & 23 & 1.3 & 1.2 \\
\hline Neodolichorhynchus & 2.9 & 1.0 & 30 & 4.3 & 1.4 \\
\hline Paratylenchus & 3.7 & 1.5 & 36 & 1.5 & 1.5 \\
\hline Psilenchus & 1.8 & 0.51 & 45 & 2.6 & 1.3 \\
\hline Tylenchorhynchus & 45.8 & 11.8 & 222 & 9.5 & 32 \\
\hline Xiphinema & 5.7 & 1.5 & 96 & 4.0 & 5.2 \\
\hline \multicolumn{6}{|c|}{ Free-living soil nematodes } \\
\hline Acrobelus & 36 & 2.5 & 221 & 8.9 & 33 \\
\hline Cephalobus & 22 & 5.6 & 110 & 3.6 & 7.5 \\
\hline Diplogaster & 1.9 & 2.5 & 33 & 2.4 & 2.1 \\
\hline Diploscapter & 1.5 & 0.5 & 22 & 1.3 & 1.2 \\
\hline Discolaimus & 1.8 & 0.4 & 39 & 2.6 & 1.7 \\
\hline Dorylaimus & 5.7 & 1.5 & 110 & 5.0 & 6.2 \\
\hline Eudorylaimus & 4.9 & 1.3 & 102 & 4.8 & 5.5 \\
\hline Laimydorus & 1.9 & 2.6 & 59 & 2.6 & 2.1 \\
\hline Mesodorylaimus & 7.6 & 2.0 & 93 & 4.2 & 6.1 \\
\hline Mononchus & 3.8 & 1.9 & 35 & 1.5 & 1.5 \\
\hline Mylonchulus & 2.2 & 1.5 & 40 & 2.0 & 1.6 \\
\hline Nygolaimus & 3.6 & 1.2 & 69 & 2.8 & 2.9 \\
\hline Panagrolaimus & 40 & 11.3 & 253 & 5.6 & 12 \\
\hline Plectus & 18.3 & 4.3 & 196 & 4.6 & 10.7 \\
\hline Rhabditis & 5.3 & 2.3 & 59 & 2.6 & 2.1 \\
\hline Rhabdolaimus & 5.8 & 1.6 & 39 & 1.2 & 1.5 \\
\hline Seleborca & 1.6 & 0.5 & 45 & 2.4 & 1.5 \\
\hline
\end{tabular}

Absolute frequency (A.F) $=$ (No of samples containing a species/ Total samples collected) 100; Relative frequency (R.F) = (Frequency of a species in a sample/ Sum of frequencies of all species present) 100; Absolute density (A.D) $=$ (No of individuals of a species in a sample/ volume of the sample) 100; Relative density (R.D) = No of individuals of a species in a sample/ Total no. of individuals in the sample ) 100; Prominence value (P.V) $=$ (Absolute density/ Absolute frequency). 


\section{Cluster analysis of rice and soybean localities}

A similarity matrix based on the proportion of shared nematode community was used to establish the level of relatedness between the different rice and soybean localities of Pakistan. Similarity of closest areas was estimated ranged from 0.64 to 0.91 . Two localities of rice Kasoor and Sahiwal (Punjab) were the closest localities for the presence of plant parasitic nematodes with the other tested localities; whereas, Nasirabad and Sukkur (Sindh) showed very low similarities $(64 \%)$ for the all nematode genera, but both localities have same nematode genus i.e., Hirschmanniella.

Similarities of nematode density obtained from quantitative data were used to create a cluster diagram. Cluster analysis, based on Nei and Li's similarity coefficients using UPGMA dendrogram, showed that all 26 rice growing localities of Pakistan could be easily distinguished based on the information generated by the community analysis of nematodes.

As expected, localities were distinguished in 9 cluster groups corresponding to the Sindh and Punjab localities, with additional sub clusters and a few independent localities (Fig. 4). In cluster analysis those areas which soil samples having abundant density of nematodes genera showing a high frequency of nematode relatedness. While observing the clustering pattern, it is evident that in most of the cases the diversity level among the localities of the rice areas in close proximity is very narrow, forming small sub-clusters with very little linkage distances. Low difference within certain locations may be due to shorter history of cultivation.

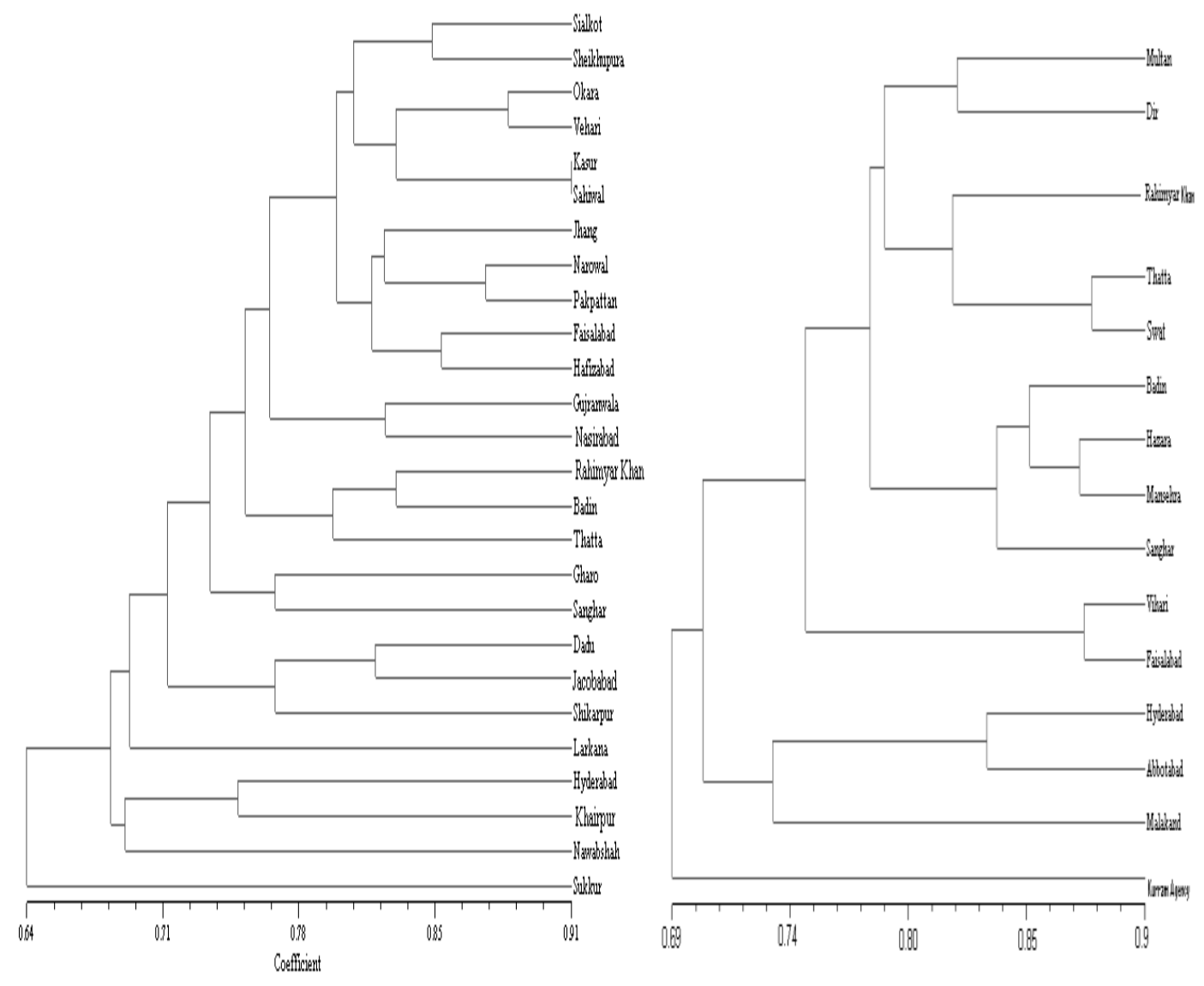

Figures 4 and 5. Dendrogram resulting from average linkage of 26 localities of rice and 15 localities of soybean growing areas of Pakistan based on nematode communities 
A similarity matrix based on the presence/absence of nematodes data was used to construct the ratio of similarity between the different soybean growing localities of three provinces of Pakistan. Similarity coefficients among various soybean growing areas of Pakistan ranged from 0.69 to 0.90 were presented.

The UPGMA cluster analysis showed that all 15 soybean growing areas could be easily distinguished based on the information generated by the presence or absence of nematodes. As expected, areas were separated into 5 distinct groups. Group A lines comprised with the similarity coefficient of 0.82 (82\%). The localities of Multan and Dir showed close similarity coefficient. Thatta and Swat 0.87 (87 \%) show more similar coefficient for the nematode incidence. Kurram Agency did not fall in any group, might be this area having dissimilar nematode fauna /or climate conditions and would have some difference of nematode density (Fig. 5).

\section{Discussion}

Nematodes co-exist together in different environment (Boag and Yeates, 1998), however, their frequency, density and diversity varies depending upon ecological and edaphic factors (Sohlenuis, 1979; Khatoon et al., 2001). Nematodes may form the most significant group for community indicator analysis because more information exists on their taxonomy and feeding groups (Gupta and Yeates, 1997) than for other macrofauna. Freeliving nematodes are very important and beneficial in the decomposition of organic material and the recycling of nutrients in soil. Nematode bacterivores and fungivores do not feed directly on soil organic matter, but on the bacteria and fungi which decompose organic matter. The presence and feeding of these nematodes speed up the decomposition process. Their feeding recycles minerals and other nutrients from bacteria, fungi, and other substrates and returns them to the soil where they are accessible to plant roots.

Detailed study has revealed that host associated nematodes were more in rice than soybean cultivated areas of Pakistan. A total of 39 genera were identified from rice while 33 genera were recorded from soybean from 41 localities throughout Pakistan. Among these nematodes, Hirschmanniella spp., was predominant and reduced yield production Tiwari and Kumar (1996) reported Hirschmanniella spp., infection in irrigated rice. Many researchers described the Hirschmanniella spp., on rice fields from various parts of the country (Maqbool and Shahina, 2001). Rose et al. (1967) reported that Tylenchorhynchus claytoni suppressed yield of soybean by $21 \%$ in microplots and Ahmad et al. (2001) studied the occurrence of plant parasitic nematodes in soybean fields of Bundel khand region. Also adding to the parasitic load were Aphelenchoides spp., Helicotylenchus spp., and Tylenchorhynchus spp., which were found in large number of samples of rice and soybean examined. However, present findings are in confirmation of the results of previous surveys carried out by many researchers that worked on similar objectives viz., Khan and Shaukat (1999), Ahmad and Nadeem (1983), Sher (1963), Robbins et al. (1982).

On the basis of trophic composition nematodes were grouped in five categories: herbivores, bacteriovores, omnivores, fungivores and predators. Herbivores representing the highest number of genera and also the highest abundance in both rice and soybean crops. While within the taxonomic groups, Hirschmanniella was the most frequently encountered genus $(21 \%)$ dominating the entire nematode community in rice and it could be related to its cosmopolitan nature of occurrence and specific host (rice). Similarly, Helicotylenchus was the most frequently occurring genus in soybean which may be due to its cosmopolitan and polyphagous nature. Aphelenchoides, Clarkus and Cephalobus 
showed comparatively high frequency and density than for other trophic group of nematodes due to mono-cropping. In rice and soybean herbivores showed highest abundance which was related to the abundance of other trophic groups. It may be concluded that a highly significant positive correlation exists between herbivores species of both rice and soybean samples. These relationships are broadly in line with findings of the Debabrata et al. (2007) and Yeates et al. (1993) who reported that free-living bacteriovorous, fungivorous and omnivorous species comprise $52 \%$ of the total nematode genera and $26 \%$ of the terrestrial genera. Higher population of herbivores may also be attributed to the fact that, the excessive use of fertilizers and other manures, add excessive nutrient to the soil. These findings are similar to the study of Tomar et al. (2006).

Cluster analysis was also performed based on nematode communities. The resulted dendogram showed that diversity level among the 26 localities of the rice areas in close proximity is very narrow, while the UPGMA cluster analysis showed that all 15 soybean growing areas could be easily distinguished. The localities of Multan and Dir showed close similarity coefficient. Thatta and Sawat showed more similar coefficient or the nematode incidence. Kurram Agency did not fall in any group and observed to be more distantly related to all groups.

\section{REFERENCES}

[1] Ahmad, R., Nadeem, A. (1983): Varietal susceptibility to rice plant nematodes. 294302pp. - In: Proceeding of $4^{\text {th }}$ National Seminar on Rice Research and Production. NARC, Islamabad.

[2] Ahmad, F., Srivastav, A.K., Pandey, R. (2001): Occurrence of plant parasitic nematodes in soybean field of Bundelkhand region. - Flora and Fauna Jhansi (7):88.

[3] Anwar, S.A., Khan, I.U. (1973): Association of nematodes with rice in Punjab. - Journal of Agricultural Research (11): 111-113.

[4] Baermann, G. (1917): Eine einfache method zur Auffindung Von Ankylostomum (Larven) in Erdproben Geneesk. - Tijdschr. Ned. Ind. 57: 131-137.

[5] Boag, B., Yeates, G.W (1998): Soil nematode biodiversity in terristerial ecosystems. Biodiversity and Concervation 7:617-630.

[6] Bridge, J., Michel, L., Plowright, R.A. ( 1990): Nematodes parasites of rice. 69- 108 pp. In: Luc, M., Sikora, R. A., Bridge, J. (eds.) Plant parasitic nematodes in subtropical and tropical agriculture, CAB International, Wallingford, UK.

[7] Cobb, N.A. (1918): Estimating the nema population of soil. Agricultural Technical Circular, US Department of Agriculture, $48 \mathrm{pp}$.

[8] Debabrata, S., Amalendu, C., Buddhadeb, M. (2007): A report on the relative abundance of Tylenchida, Dorylaimida and Mononchida population of guava orchards in 24paraganas (South), West Bengal, India. - Indian Journal of Nematology (37):72-77.

[9] Ettema, C.H. (1998): Soil nematodes diversity: Species coexistence and ecosystem. Journal of Nematology 30(2):159-169.

[10] Good, J.M. (1973): Nematodes. 527-543 pp. - In: Caldwell, B.E. (ed) Soybeans: Improvement, production and uses., American Society of Agronomy.

[11] Gupta, V.V.R., Yeates, G.W. (1997): Soil microfauna as bioindicators of soil health. 201234 pp. - In: Doube, B.M., Gupta, V.V.R. (eds) Biological Indicators of Soil Health., $\mathrm{CAB}$ International, Wallingford, UK.

[12] Khan, A., Bilqees, F.M. (1994): Plant parasitic nematodes associated with paddy crops in Bajore Agency, Pakistan. 1-3 pp. - Proceeding of Pakistan Congress of Zoology, University of Karachi, Karachi. 
[13] Khan, A., Shaukat, S.S. (1999): Survey of stylet bearing nematodes associated with rice (Oriza Sativa L.) in Thatta district, Sindh, Pakistan. - Sarhad Journal of Agriculture (15): 221-225.

[14] Khatoon, M., Sharma, S., Saxena, S.K. (2001): Community analysis of predatory nematodes of Rohilkhand division, UP. - Current Nematology (12): 11-14.

[15] Maqbool, M.A. (1981): Occurrence of root-knot and cyst nematodes in Pakistan. Nematologia Mediterranea (9): 211-212.

[16] Maqbool, M.A. (1983): Description of Paktylenchus tuberosus gen. n., sp.n. (Nematoda: Tylenchinae) from Pakistan. - Journal of Nematology (15):76-79.

[17] Maqbool, M.A. (1984): Nematode diseases of rice in Pakistan and their control. National Nematological Research Centre, University of Karachi, Urdu version.

[18] Maqbool, M.A., Hashmi, S. (1982): Nematode associated with Oryza sativa L. in Pakistan. - $21^{\text {st }}$ Annual Meeting of Society of Nematologists, 26-30 July, 1982. Knoxuille, Tennesse, USA.42 pp.

[19] Maqbool, M.A., Shahina, F. (2001): Systematics and distribution : Biodiversity of nematode fauna in Pakistan. - National Nematological Research Centre, University of Karachi,Karachi-75270, Pakistan.179 pp.

[20] Musarrat, A.R., Firoza, K., Shahina, F. (2006): Study of root-knot nematodes in NWFP and Sindh, Pakistan. - Pakistan Journal of Nematology (24):1-7.

[21] Nei, N., Li, W. (1979): Mathematical model for studing genetics variation in term of restriction endonucleases. - Acta Hortica (262): 305-310.

[22] Norton, D.C. (1978): Ecology of plant parasitic nematodes. - John Wiley and Sons, New York, $268 \mathrm{pp}$.

[23] Robbins, R.T., Riggs, R.D., Von Steen, D. (1982): Phytoparasitic nematodes surveys of Arkansas cotton fields, 1986-1988. - Journal of Nematology (21): 619-623.

[24] Rohlf, F.J. (2005): NTSYS-pc. Numericaltaxonomy and multivariate analysis system. Exeter Software, New York.

[25] Sasser, J.N., Freckman, D.W. (1987): Aworld perspective of Nematology: The Role of the Society. 7-14 pp. - In: Veech, J.A., Dickson, D.W. (eds.) Vistas on Nematology, Society of Nematologists, Hyattsville, Maryland, USA.

[26] Schmitt, D.P., Noel,G.R. (1984): Nematode parasites of soybean. 13-59 pp. - In: Nickel, W.R. (ed.) Plant and Insect Nematodes. - Marcel Dekker, New York.

[27] Shahina, F., Zarina, B., Firoza, K., Soomro, M.H., Javed, N. (2009): Research on rootknot nematodes, Meloidogyne by Pakistani scientists: A review with description of species prevalent in Pakistan. - Pakistan Journal of Nematology (27):1-115.

[28] Sher, S.A. (1963): A revision of the Hoplolaimus (Nematoda) II. Hoplolaimus Daday, 1905 and Aorolaimus n.gen. - Nematologica (9):267-295.

[29] Sohlenuis, B. (1979): Acarbon budget for nematodes, rotifers and tardirades in a Swedish coniferous forest soil. - Holarctic Ecology (2):30-40.

[30] Timm, R.W. (1955): The occurrence of Aphelenchoides besseyi Christie, 1942 in deep water paddy of East Pakistan. - Pakistan Journal of Science (7):47-49.

[31] Timm, R.W. (1956): Nematode parasites of rice in East Pakistan. Pakistan Review of Agriculture (2): 115-118.

[32] Tiwari, S.P., Kumar, S. (1996): Root knot nematode Hirschmanniella Oryza (Sottwedel, 1889) Lucand Goodey, 1963 associated with Cyperus (L.)Species in some rice growing districts of Madhya Pradesh, India. - Weed News (3):9-14.

[33] Tomar, V.V.S., Baniyamuddin, M., Ahmad, W. (2006): Community structure of soil inhabiting nematodes in a mango orchard at Aligarh, India. - International Journal of Nematology (16): 89-101.

[34] Yeates, G.W., Bongers, R.G.M., Goede, R.G.M., Freckman, D.W., Georgieva, S.S. (1993): Feeding habits in soil nematodes families and genera an outline for soil ecologists. - Journal of Nematology 25: 315-331. 